\title{
La teoría fundamentada en educación matemática
}

\section{Grounded theory in mathematics education}

DOI: $10.46932 / \mathrm{sfjdv3n1-086}$

Received in: Jan 30st, 2021

Accepted in: Feb 1th, 2022

\section{Ana Rosa Flores Valdez}

-Ingeniera Civil por la Universidad Autónoma del Estado de México (UAEM)

-Maestra en Investigación de la Educación por el Instituto Superior de Ciencias de la Educación del

Estado de México (ISCEEM)

-Estudiante del programa Doctoral de Ciencias de la Educación en el Instituto Superior de Ciencias de la Educación del Estado de México (ISCEEM)

Instituto Superior de Ciencias de la Educación del Estado de México (ISCEEM)

Ex Rancho Los Uribe s/n, Santa Cruz, Atzcapotzaltongo, 50030 Toluca de Lerdo, Méx.

E-mail: ana.flores@isceem.edu.mx

\section{RESUMEN}

En este artículo se presenta un avance de la investigación que se está realizando en el Programa de Doctorado del Instituto Superior de Ciencias de la Educación del Estado de México (ISCEEM), denominada "Construcción de la Inferencia científica de los Estudiantes de Bachillerato en la resolución de problemas contextualizados". En el presente documento se aborda una aplicación de la Teoría Fundamentada en el campo de la investigación en Educación matemática, tomando la información que se ha recopilado de la investigación mencionada.

Palabras clave: Teoría fundamentada, Educación matemática.

\begin{abstract}
The paper below presents a research advance being carried out in the Doctoral Program in the Instituto Superior de Ciencias de la Educación del Estado de México (ISCEEM), wich call "Construction of the Scientific Inference of High School Students in the resolution of contextualized problems". This article presents a Grounded Theory application in Mathematical education field, considering information that has been collected in the aforementioned research.
\end{abstract}

Keywords: Grounded theory, Mathematical education.

\section{INTRODUCCIÓN}

Kilpatrick (1998) menciona que el campo de estudio de la Educación matemática (EM) surge hacia el final del siglo XIX como respuesta de las Universidades de distintos países ante la problemática derivada de la necesidad de contar con docentes mejor preparados para la enseñanza de la Matemática. Por su parte, Ernest (2016) considera a la EM como un campo de estudio multidisciplinario que relaciona 
diversas áreas: la naturaleza propia de las Matemáticas, el aprendizaje y su enseñanza, así como el contexto social que la rodea y sus aplicaciones.

Como en cualquier campo en el que se realice alguna investigación, el investigador dispone de dos tradiciones para desarrollar la parte metodológica de su trabajo: la aristotélica y la galileana, el Verstehen y el Erklären, la comprensión y la explicación. La primera busca dar cuenta del telos del evento estudiado, con explicaciones (llamadas teleológicas) que "aclarasen 'con el fin de que' ocurrían los fenómenos" (Mardones \& Ursúa, 1982, pág. 17); se busca, de esta manera, la comprensión.

Por otro lado, la tradición galileana es considerada por Mardones \& Ursúa (1982) como la explicación científica del fenómeno, en función de leyes que relacionan los fenómenos de manera matemática, en donde la comparación entre la hipótesis y las consecuencias que han sido deducidas de la observación del evento dictarán su valor explicativo.

Estas tradiciones han sido asociadas a dos maneras de hacer investigación: la cualitativa y la cuantitativa. Estas son denominadas por Salomon (1991) como aproximaciones metodológicas, la primera denominada también analítica, y la segunda sistémica. El choque entre ambas ha provocado lo que se ha definido como un "debate cualitativo-cuantitativo" (Salomon, 1991, pág. 10).

Aún con la existencia de este debate, es importante señalar que ambas perspectivas permiten una multiplicidad de visiones necesarias para poder entender los fenómenos que se originan dentro de la EM; por ello es necesario reconocer que el campo que estudia los eventos de enseñanza y aprendizaje de las Matemáticas no se encuentra restringido de manera exclusiva a alguna de estas tradiciones.

En este artículo se presenta un enfoque teórico cualitativo orientado a la EM, mostrando que la tradición del Verstehen puede dar una visión de los fenómenos estudiados en este campo, y que no se limita a la tradición del Erklären.

\section{MÉTODO}

Esta investigación se desarrolla dentro del campo de la EM, teniendo como sustento metodológico a la investigación cualitativa, y a la Teoría fundamentada (TF) como enfoque teórico. Esta surge en 1967, con la publicación del libro The Discovery of Grounded Theory de Glaser \& Strauss, en donde señalan que buscan "promover el descubrimiento de la teoría a partir de los datos obtenidos y analizados sistemáticamente en la investigación social” (Glaser \& Strauss, 1967, pág. 1).

Hoy en día se conocen cuatro tendencias de este enfoque: la de Glasser publicada en 1978, Theoretical Sensivity; la desarrollada en 1990 por Strauss\&Corbin, en Basics of Qualitative Research; la tendencia de Charmaz presentada en el 2000 en Grounded theory methodology: Objectivist and 
constructivist qualitative methods; y finalmente, la de Clarke contenida en la publicación de su trabajo en el 2005, Situational analysis: Grounded theory after the postmodern turn, (Teppo, 2015).

Dentro de la EM una propuesta interesante se presenta en el trabajo de Vollsted (2015), en donde toma como fundamento teórico-metodológico la TF desde la perspectiva de Strauss \& Corbin (1998) y la aplica al campo de la EM. A continuación, se presenta el desarrollo de la TF empleando información derivada de la investigación "Construcción de la Inferencia científica de los estudiantes de Bachillerato en la resolución de problemas contextualizados" que se está desarrollando actualmente en el Programa doctoral del ISCEEM.

\section{RESULTADOS}

Es importante resaltar que lo aquí presentado se hace de manera sucinta, con elementos básicos de la TF en EM; sin embargo, es preciso señalar que esta representación teórica es compleja, porque requiere una profunda minuciosidad, además de un amplio trabajo de campo; sin embargo, su complejidad se ve recompensada con la valiosa información que se obtiene desde la perspectiva desde la cual se analiza el fenómeno estudiado.

Inicialmente podrá parecer que el estudio empírico da pocas luces sobre las decisiones que se han de tomar durante el desarrollo de la investigación, los datos pueden verse confusos y probablemente no sea clara ni evidente alguna relación entre ellos; sin embargo, desarrollar una TF de manera empírica que combine tanto aspectos metódicos como metodológicos, brindará pautas necesarias durante el proceso de investigación, (Vollsted, 2015).

El primer punto planteado por Vollsted (2015) es el de Antecedentes y enfoque del estudio. Este señala la importancia que tiene el enfocarse en los sujetos de la investigación. En el caso de la investigación a la que se hace referencia, habrá que centrarse en aspectos que coincidan con sus objetivos, por ejemplo: los elementos que intervienen en la construcción de la inferencia científica por parte de los estudiantes, cuando estos se enfrentan a la resolución de un problema contextualizado. No se debe olvidar que el énfasis está en la perspectiva de los alumnos.

Además, en el caso del objetivo anterior, el investigador deberá cuestionarse lo que es, en primera instancia, un problema contextualizado, qué es la inferencia científica y qué significa una resolución de problemas. Es en este momento cuando se debe hacer una investigación teórica sobre estas cuestiones con el fin de delimitar conceptualmente los términos.

La segunda etapa propuesta por Vollsted (2015) es denominada Realización del estudio. Al inicio de la investigación con TF "no existe una teoría completa sino, por el contrario, es un campo de estudio abierto cuyos aspectos relevantes se vuelven cada vez más claros a lo largo del proceso de investigación" 
(Vollsted, 2015, pág. 25). El investigador no sabe lo que va a hallar, por ello debe ser cuidadoso en recabar la mayor información posible.

En la investigación sobre Inferencia científica, se ha recopilado una gran cantidad de material de audio, video, imágenes, chats y cuestionarios en línea. Se han desarrollado dos series de entrevistas individuales a cada estudiante. En la primera de ellas se tuvo el acercamiento (virtual) con ellos, estas revelaron, por ejemplo, información sobre el contexto inmediato del alumno (escolar y familiar) y sus actitudes ante las actividades escolares, entre otras. La segunda etapa de entrevistas, también virtual, recuperó elementos sobre la manera en la cual el alumno resuelve problemas matemáticos. Cada entrevista se acompañó de notas de campo.

La siguiente etapa de la propuesta de Vollsted (2015) corresponde a lo que ha denominado Sensibilidad teórica y conceptos sensibilizadores. Se parte de las consideraciones de que no hay ninguna hipótesis que deba probarse, y de que no existe una teoría desarrollada por completo en el campo en el cual se está investigando. De esta manera será necesario que, para el desarrollo de la TF, el investigador recurra a lo que se denomina 'sensibilidad teórica'.

Esta sensibilidad teórica requiere que se emplee el conocimiento que ya se posee sobre el tema. El investigador no debe, ni puede, desecharlo, porque en última instancia no se puede hacer a un lado lo que somos, lo que implica el no poder separarse de lo que se sabe; sin embargo, se debe tener cuidado en cómo se usa tal conocimiento. Strauss \& Corbin (1998) señalan que ese bagaje que el investigador posee puede ser traído al tratamiento de los datos; pero de una manera consciente y sistemática.

La etapa cuarta en el desarrollo de la TF es nombrada por Vollsted (2015) como Interdependencia de la recopilación de datos, el análisis y el desarrollo de la Teoría. Aquí, los datos que han sido recopilados serán analizados de manera ordenada y sistemática, esto permitirá que gradualmente se pueda descubrir, desarrollar y verificar la teoría. Aquí surge la interrelación entre esta, los datos descubiertos y su análisis.

Con la información sobre la investigación de Inferencia científica anteriormente citada, si se hubiesen dejado entrevistas sin analizar a la vez que se recopilaban otras, podría pasarse por alto el hecho de que tal vez varios de los entrevistados señalaban de manera recurrente información sobre su estado de ánimo, la cual puede ser parte importante en la construcción de una inferencia científica.

Esa información es relevante, aunque no se haya considerado de inicio, si se dejase pasar de largo, podría suceder que la investigación no cuente con una pieza importante, y que, en caso de identificarla posteriormente, el acceso a campo y el acercamiento con los estudiantes sea imposible, de esa manera se perdería información fundamental. El investigador debe estar atento a los datos y lo que estos revelen, aunque sean insospechados al inicio de su trabajo. 
Retornando a la interdependencia entre datos, análisis y teoría, esta es conocida como 'muestreo teórico', y descansa sobre conceptos que durante el análisis demostraron relevancia teórica. Esto se observa cuando los conceptos aparecen de manera reiterada en los datos o, incluso, si se encuentran notablemente ausentes al comparar los incidentes observados, (Vollsted, 2015).

Strauss \& Corbin (1998) señalan que a medida que avanza el trabajo de investigación, la sensibilidad aumenta, de manera que es recomendable echar de nuevo un vistazo a los datos que ya se han codificado, porque probablemente ese conocimiento adicional con el que se cuenta (debido al propio proceso analítico que se ha hecho en la investigación) permita recuperar la información que no se vio o no se consideró relevante en el primer análisis.

Es natural que el investigador pueda sentirse abrumado ante tanta información recabada, porque resulta un verdadero desafío tomar la decisión de por dónde se comenzará con el análisis de los datos. En la investigación de la Inferencia científica que está sirviendo como apoyo en este artículo, una parte de la información recopilada proviene de entrevistas (en línea) con estudiantes de Bachillerato General (BG), las cuales fueron acompañadas con notas (memos), tal como se muestra en el ejemplo siguiente:

Se comenzó por el análisis de las entrevistas, se transcribieron de manera detallada, cuidando de registrar el minuto en el cual se realizaba alguna pregunta, también si se presentaba algún aspecto relevante en misma. Se muestra a continuación un segmento de la entrevista con una alumna a quien llamaremos Victoria, por cuestiones de confidencialidad.

Tabla 1 Ejemplo de transcripción de entrevista

\begin{tabular}{ll}
\hline \multicolumn{1}{c}{ Tiempo } & \multicolumn{1}{c}{ Aspectos principales } \\
$(\ldots)$ & $(\ldots)$ \\
& -Investigadora: Tú ¿cómo te la estás pasando ahorita con la pandemia y todo esto? \\
& -Victoria: Pues al principio era de las personas que decían que no me afectaba; pero \\
pues sí, empiezas a notar cambios, más que todo en nuestro comportamiento, ¿no?; \\
bueno yo lo veo así, llegó a un punto donde me enojaba todo y ya no aguantaba a \\
\\
nadie, bueno de mi familia; pero ya creo que lo estoy llevando mejor. \\
$(\ldots)$
\end{tabular}

Nota. En la TF es sumamente importante contar con toda la información posible. Una forma de hacerlo es con el análisis de entrevistas, por lo que es necesario transcribir toda la información que el sujeto colaborador nos proporcione.

Esta serie de entrevistas a los estudiantes llevó a considerar en la investigación la relevancia que puede tener el estrés cuando resuelven un problema y construyen sus inferencias. De manera inicial no se había contemplado esta información, que fue recurrente; pero una vez conocida hizo que se identificara el código de estrés para clasificarla. Fue necesario, además, que se hiciera una investigación teórica sobre el tema, para poder identificar otros datos que calificasen como tal dentro de las demás entrevistas.

En el análisis de la información, Strauss \& Corbin (1998) recomiendan estar pendientes de la saturación teórica. Esta se presenta cuando ya no se desarrollan categorías nuevas en los datos con los que se cuenta, por lo que la relación que existe entre las categorías parece estar establecida y validada. En este 
punto ya no se recopila más información y se escribe la teoría tal como se desarrolla hasta entonces. El investigador podrá identificar este momento, cuando en la información recopilada ya no surge un dato relevante que lo lleve a una nueva categoría, es decir, ya no se encuentra información nueva.

El siguiente paso es nombrado por Vollsted (2015) como Análisis de datos. Es en esta etapa cuando se procede a la codificación. Strauss \& Corbin (1998) proponen tres tipos de codificación: abierta, axial, y selectiva. Este proceso generalmente alterna entre la codificación abierta y la codificación axial, aquí el análisis está marcado por una interacción continua entre los supuestos planteados sobre la relación existente entre los fenómenos, y su intento de verificación con respecto a los datos, lo que sirve de fundamento empírico a la TF, Teppo (2015).

En lo que respecta a los tipos de codificación, Strauss \& Corbin (1998) señalan que la codificación abierta es un proceso de tipo analítico, a través del cual se identifican los conceptos que permiten descubrir propiedades y dimensiones de los datos. Mientras que la codificación axial permitirá relacionar categorías con sus respectivas subcategorías; el término 'axial' se refiere a que esta codificación se da alrededor de una categoría (la cual constituye el 'eje'), lo que permite enlazar las categorías de acuerdo con sus propiedades y dimensiones, y refinar de esta manera la teoría.

Un ejemplo de estas codificaciones se presenta a continuación, con una sesión de trabajo para que el estudiante resuelva problemas matemáticos. Esta sesión fue de manera virtual, se presenta la información del alumno al que llamaremos Hugo:

Tabla 2 Ejemplo de codificación

\begin{tabular}{|c|c|}
\hline 1. Investigadora: & $\begin{array}{l}\text { Perfecto. Ahora, nos está diciendo que se trata de la Tierra; pero nosotros no estamos viendo } \\
\text { la tierra, ¿correcto? }\end{array}$ \\
\hline 2. Hugo: & Sí, es correcto \\
\hline 3. Investigadora: & ¿qué estamos viendo? \\
\hline 4. Hugo: & La ciudad de Alejandría \\
\hline 5. Investigadora: & $\begin{array}{l}\text { Ajá, ok, y ahora, antes de pasar a cualquier cosa, lo que les decía, ¿tenemos todos los datos } \\
\text { ahí?, ¿o nos hace falta escribir o trazar algún dato? }\end{array}$ \\
\hline 6. Hugo: & $\begin{array}{l}\text { Mmm ( } 5 \text { seg) No, pues sí tenemos todo. Tenemos la medida de los ángulos, que trae las líneas } \\
\text { paralelas de los rayos del sol }\end{array}$ \\
\hline 7. Investigadora: & Ok, a ver, léelo y compáralo con el esquema \\
\hline \multirow[t]{2}{*}{ 8. Hugo } & $\begin{array}{l}\text { ( } 4 \mathrm{seg} \text { ) [murmura el problema] Pues siento que el único dato que nos faltaría en la gráfica son } \\
\text { los } 158 \mathrm{~km} \text { que hay entre Alejandría y Siena. }\end{array}$ \\
\hline & $(\ldots)$ \\
\hline
\end{tabular}

Nota. En la Teoría fundamentada es necesario recabar toda la información posible, incluyendo los silencios. La etapa de codificación permite identificar aquellos códigos que van saltando a la vista, por ejemplo, en la codificación (8) se observa que a Hugo comienza a construir una hipótesis explicativa, lo que, en el desarrollo de la investigación sobre Inferencia científica, forma parte de la sensibilidad teórica del investigador ya que se ha investigado sobre los tipos de inferencia para poder identificarlos en la información que se recupera.

Al hacer un análisis a los datos, se identifica que Hugo comienza a construir un tipo de inferencia denominado Abducción, la cual le permitirá introducir una nueva idea (esta información se conoce porque se ha investigado sobre los tipos de inferencia, esto constituye parte del marco teórico de la investigación 
mencionada, el cual no se mostrará en el artículo, debido a la amplitud de este y a que queda fuera del objetivo del presente trabajo).

Con esta información se genera el código Abducción, teniéndolo presente, se incorporarán bajo este, los incidentes que califiquen como tal, y en una fase posterior de la investigación, con él se podría desarrollar el primer concepto.

Teppo (2015, pág.7) sostiene que los registros que surgen de la investigación se activan en el momento en que un dato presenta cierto aspecto que llama la atención del investigador. Estos surgen cuando una idea conceptual se deriva de un aspecto particular del dato, lo que "promueve una conceptualización que es más apropiadamente fundamentada en los datos”. En el ejemplo presentado, el registro se activa cuando Hugo comienza a introducir una idea, porque la Abducción desde el sentido inferencial sugiere que algo puede ser, (Gelfert, 2014).

No se debe olvidar que la TF se desarrolla sobre datos empíricos, Vollsted (2015, pág.4) señala que lo que se busca es "descubrir elementos de una teoría sobre nuestra pregunta de investigación en estos datos", por ello el investigador debe partir de expresiones concretas (que hayan sido obtenidas de entrevistas y de la codificación) para poder arribar a una idea más general, que pueda resultar interesante y que trate de dar respuesta a la pregunta de investigación.

Las categorías principales y sus subcategorías describen los indicadores que ocurren de manera frecuente en los datos de la investigación, estos forman un patrón que permitirá lograr una teoría que describa al fenómeno que se estudia, Vollsted (2015) señala que es en este momento cuando termina la aplicación de la TF.

Finalmente, Vollsted (2015) señala que la idea fundamental en el desarrollo de teoría mediante la TF es la obtención de todas las ideas que se encuentren detrás de los datos recuperados de lo que expresan los sujetos entrevistados, la construcción de hipótesis sobre la relación que se genera en las ideas que vamos formulando, y finalmente, reafirmar o desechar estas relaciones o vínculos. Al final de todo este proceso se llega a una teoría que se encuentra fundamentada empíricamente.

\section{CONCLUSIONES}

El Verstehen de la investigación cualitativa aporta la comprensión de un fenómeno desde una perspectiva que puede complementar la mirada del Erklären de la cuantitativa. Por su parte la investigación en Educación matemática no está limitada a ser investigada por una o por otra tradición, de hecho, la Teoría fundamentada como representación teórica cualitativa puede emplearse para estudiar los fenómenos en el campo. 
Esta representación puede ser flexible sin perder rigor, de manera que la aplicación de esta depende también de la creatividad y la sensibilidad del investigador, quien no podrá negar su propia esencia, experiencia y conocimientos previos, los cuales pueden ayudar al desarrollo de la Teoría fundamentada si se emplean de manera consciente y teniendo siempre presente el objetivo del trabajo de investigación.

Los datos serán siempre importantes, tanto los verbales como los no verbales. En la Teoría fundamentada todo importa, incluyendo las percepciones y los registros del investigador, quien deberá desarrollar la habilidad de registrar toda la información, que tal vez a primera vista no sea relevante; pero que sin duda podrá ser de utilidad conforme se avance en la investigación.

Finalmente, esta investigación sostiene que cualquiera de las dos tradiciones: cualitativa o cuantitativa, puede aportar información que permitirá complementar de manera integral un fenómeno desarrollado en el campo de la Educación matemática. 


\section{REFERENCIAS}

ACRIVIRC. (2020). Cusco.

Álvarez, M. (2014). Manual para elaborar Manuales de Políticas y Procedimientos. Mexico D.F.: Panorama Editorial S.A.

Angulo, N. (1 de abril de 2009). ¿Qué son los observatorios y cuáles son sus funciones? Obtido de Innovación Educativa: http://www.redalyc.org/pdf/1794/179414895002.pdf

BCE. (Abril de 2020). REPORTE DE COYUNTURA SECTORIAL AGROPECUARIO. 48. Obtido em 01 de Enero de 2022, de https://contenido.bce.fin.ec/documentos/PublicacionesNotas/Catalogo/Encuestas/Coyuntura/Integradas/ etc201904.pdf

Bernis, J. M., \& Pàmies, C. B. (2004). VARIEDADES Y MEJORA DEL ARROZ (Oryza sativa, L.) (Universidad Internacional de Cataluña ed.). UIC - Campus de l'Ebre. Obtido de http://espacio.uned.es/fez/eserv/bibliuned:UNEDCentroAsociadoTortosa-Libros-

5025/Franquet_Bernis_JoseMaria_Variedades.pdf

Bioenciclopedia. (2021). Obtido de Bioenciclopedia: encontrado https://www.bioenciclopedia.com/vicuna/

Castellanos, F. (19 de Julio de 2021). El Universo. Obtido em 04 de 01 de 2022, de https://www.eluniverso.com: https://www.eluniverso.com/noticias/ecuador/con-un-precio-de-28-porsaca-de-210-libras-de-arroz-en-cascara-productores-reciben-una-ganancia-de-380-por-hectarea-en-treso-cuatro-meses-nota/

CFN. (Julio de 2021). /www.cfn.fin.ec. Obtido em 12 de Enero de 2022, de https://www.cfn.fin.ec/wpcontent/uploads/downloads/biblioteca/2021/fichas-sectoriales-3-trimestre/Ficha-Sectorial-Arroz.pdf

Chang, J. V. (2008). CULTIVO DE ARROZ SISTEMA INTENSIFICADO SICA-SRI EN ECUADOR. ADMICORPORACION, 5.

CONQUITO. (12 de MARZO de 2017). CONQUITO. Obtido de QuitoTech un portal que hace de Quito una ciudad digital y emprendedora: http://www.conquito.org.ec/

Ernest, P. (mayo de 2016). The unit of analysis in mathematics education: bridging the political-technical divide? Educational Studies in Mathematics, 92(1), 37-58. doi:https://doi.org/10.1007/s10649-016-96894

Fontagro. (2019). fontagro. Obtido em 07 de 01 de 2022, de Fontagro: https://www.fontagro.org/new/proyectos/arrozmasproductivo/es

Gelfert, A. (marzo de 2014). Observation, Inference, and Imagination: Elements of Edgar Allan Poe's Philosophy of Science. (S. Erduran, Ed.) Science \& Education, 23, 589-607. Obtido de https://link.springer.com/article/10.1007/s11191-012-9551-8

Glaser, B. G., \& Strauss, A. L. (1967). The Discovery of Grounded Theory. EEUU: Aldine Transaction. 
IICA. (10 de Octubre de 2017). Proyecto Cultivar más con menos: Adaptación, validación y promoción del Sistema Intensivo del Cultivo Arrocero (SRI) en las Américas como una respuesta al cambio climático. 5. Obtido em 06 de 01 de 2022, de http://sri.ciifad.cornell.edu/spanish/Taller_Regional_SRI_101017.pdf

Kilpatrick, J. (1998). La investigación en educación matemática: su historia y algunos temas de actualidad. Em J. Kilpatrick, P. Gómez, \& L. Rico (Edits.), Educación Matemática (pp. 1-18). Bogotá: Grupo Editorial Iberoamérica.

Mardones, J. M., \& Ursúa, N. (1982). Filosofía de las Ciencias Humanas y Sociales. Barcelona: Fontamara.

Mendoza, D. A. (2017). Guía para el establecimiento y monitoreo del cultivo de arros bajo la metodologia SRI. FONTAGRO, 7. Obtido em 05 de 01 de 2021

Mincetur. (2010). Perfil de Mercado y Competitividad Exportadora de Prendas de Vestir de Vicuña. Lima.

Quijije, B. A., Carvajal, S. J., Garcia, K. E., \& Cedeño, W. B. (04 de 03 de 2019). Costo, volumen y utilidad del cultivo de arroz, cantón Samborondón (Ecuador). Espacios, 40(7), 10. Obtido de http://www.revistaespacios.com/a19v40n07/a19v40n07p16.pdf

Rodriguez, R. L. (2010). SITUACION ARROCERA ECUATORIANA. CORPORACIÓN DE INDUSTRIAS ARROCEROS DEL ECUADOR, 4. Obtido em 04 de 02 de 2022, de http://www.acpaarrozcorrientes.org.ar/Jornadas-2010/11.pdf

Salomon, G. (1991). Transcending the Qualitative-Quantitative Debate: The Analytic and Systemic Approaches to Educational Research. Educational Researcher, 20(6), 10-18.

Shiguango, M. F. (25 de Agosto de 2014). COMPARACIÓN AGRONÓMICA DE OCHO CULTIVARES DE ARROZ EN DOS TIPOS DE SUELO BAJO EL MÉTODO SRI. (F. Eison Valdiviezo, Ed.) 14. Obtido em 05 de 01 de 2022

Strauss, A., \& Corbin, J. (1998). Basics of Qualitative Research : Techniques and Procedures for Developing Grounded Theory (2a ed.). EEUU: SAGE Publications.

Teppo, A. R. (2015). Grounded Theory Methods. Em A. Bikner-Ahsbahs, C. Knipping, \& N. Presmeg (Edits.), Approaches to Qualitative Research in Mathematics Education (pp. 3-22). Springer. doi:10.1007/978-94-017-9181-6

Uphoff, N. (22 de Julio de 2015). SISTEMA DE INTENSIFICACIÓN DEL CULTIVO DEL ARROZ (SRI). IICA, 3. Obtido em 06 de 01 de 2022 de http://sri.ciifad.cornell.edu/spanish/IICA_SRI_Preguntas_Frecuentesz_Uphoff042916.pdf

Vollsted, M. (2015). To See the Wood for the Trees: The Development of Theory from Empirical Interview Data Using Grounded Theory. Em A. Bikner-Ahsbahs, C. Knipping, \& N. Presmeg (Edits.), Approaches to Qualitative Research in Mathematics Education (1a ed., pp. 23-48). Springer. 